\title{
On Some Characterization of Preinvex Fuzzy Mappings
}

\author{
Muhammad Bilal Khan ${ }^{1, *}$, Muhammad Aslam Noor $^{2}$ and Khalida Inayat Noor ${ }^{3}$
}

1 Department of Mathematics, COMSATS University Islamabad, Pakistan e-mail: bilal42742@gmail.com

2 Department of Mathematics, COMSATS University Islamabad, Pakistan e-mail: noormaslam@gmail.com

3 Department of Mathematics, COMSATS University Islamabad, Pakistan e-mail: khalidanoor@hotmail.com

\begin{abstract}
In this paper, a new notion of generalized convex fuzzy mapping is introduced, which is called $\alpha$-preinvex fuzzy mapping on the $\alpha$-invex set. We have investigated the characterization of preinvex fuzzy mappings using $\alpha$-preinvex fuzzy mappings, which can be viewed as a novel and innovative application. Some important and significant special cases are discussed. We have also investigated that the minimum of $\alpha$-preinvex fuzzy mappings can be characterized by fuzzy $\alpha$-variational like inequalities.
\end{abstract}

\section{Introduction}

The concept of invex set was firstly introduced by Israel and Mond [2], in 1986. Dealing with convexity they made a comparative study that investigated some different properties of invex sets. In convex set $K \subseteq \mathbb{R}^{n}$, the path $(1-\tau) u+\tau y=u+\tau(y-u)$ which is contained in $K$, reflect that the starting and end point of path is $u$ and $y$, respectively. If $(y-u)=\xi(y, u)$, then convex set becomes invex set $K \subseteq \mathbb{R}^{n}$ with respect to bi-function $\xi: K \times K \rightarrow \mathbb{R}^{n}$ because $u+\tau(y-u)=u+\tau \xi(y, u)$. But the converse is not true because the path essentially says that path starting from $u$ which is included in $K$ and do not need that $y$ have to be one of the end point of the path which is contained in $K$ convexity. Convexity plays an essential role in many areas of mathematical analysis and due to its vast applications in diverse areas, many authors

Received: June 1, 2020; Accepted: July 7, 2020

2010 Mathematics Subject Classification: 49J40.

Keywords and phrases: preinex fuzzy mappings, $\alpha$-preinvex fuzzy mappings, $\alpha \xi$-monotone fuzzy operators, fuzzy $\alpha$-variational-like inequalities, fuzzy optimization.

* Corresponding author

Copyright $(2) 2021$ Authors 
extensively generalized and extended this concept using novel and different approaches. In [9], Hasnon introduced a useful generalization of convex function that is invex function and proved the validity of significant results that hold both for convex and invex function under few conditions. Israel and Mond [2], introduced the concept of preinvex functions on the invex sets. Later on Mohan and Neogy [12], further extended their work by proving that subject to certain conditions a preinvex function defined on the invex set is invex function and vice versa and they also showed that quasi-invex function is quasipreinvex function. Noor [18], and Weir and Mond [28], showed that the preinvex functions preserved some important properties of convex functions. Furthermore, Noor [15], and Yang and Chen [30], studied the optimality conditions of differentiable preinvex functions on the invex set that can be characterized by variational inequalities. Another class of $\alpha$-preinvex mapping on the $\alpha$-invex set (non-convex function) was introduced by Jeyakumar and Mond [10], that have a significant applications in generalized convex programming and multiobjective optimization. In [5], Chang and Zadeh introduced the fuzzy mappings. Many authors, Furukawa [7], Nanda, and Kar [13], and Syau [27], worked on the concept of fuzzy mapping from $\mathbb{R}^{n}$ to the set of fuzzy numbers, Lipschitz continuity of fuzzy valued, fuzzy logarithmic convex and quasiconvex fuzzy mappings. Based on the concept of ordering illustrated by Goetschel and Voxman [8], Yan and $\mathrm{Xu}$ [31], presented the concepts of epigraphs and convexity of fuzzy mappings, and described the characteristic of convex fuzzy and quasi-convex fuzzy mappings. The idea of fuzzy convexity has been generalized and extended in diversity of directions, which has significant implementation in many areas. It is worthy to mention one of the most considered generalization of convex fuzzy mapping is preinvex fuzzy mapping. The idea of fuzzy preinvex mapping on the fuzzy invex set was introduced and studied by Noor [14], and verified that a fuzzy optimality conditions of differentiable fuzzy preinvex mappings can be distinguished by variational-like inequalities. Moreover, any local minimum of a preinvex fuzzy mapping is a global minimum on invex set and necessary and sufficient condition for fuzzy mapping is to be preinvex if its epigraph is an invex set. In [22], Syau further modified the concept of preinvex fuzzy mapping that was presented by Noor [14]. Syau and Lee [26], also discussed the terminologies of continuity and convexity through linear ordering and metric defined on fuzzy numbers. Extension in the Weirstrass Theorem from real-valued functions to fuzzy mappings is also one of their significant contribution in the literature. Li and Noor [11], established an equivalence condition of preinvex fuzzy mapping and characterizations about preinvex fuzzy mappings with some conditions. With the support of examples, $\mathrm{Wu}$ and $\mathrm{Xu}$ [29], 
modified the concept of convex fuzzy mappings and designed new approach regarding existence of a fuzzy preinvex mapping under the condition of lower or upper semicontinuity. In 2012, Rufian-Lizana et al. [19], reviewed the presented literature and made necessary amendments in the results presented by $\mathrm{Wu}$ and $\mathrm{Xu}$ [29], about invex fuzzy mappings. Rufian-Lizana et al. [20], provided the necessary and sufficient condition for differentiable and twice differentiable preinvex fuzzy mappings. With the help of examples, they proved validity of characterizations and improved previous result given with the help if some conditions by $\mathrm{Li}$ and Noor [11]. For further study of literature, we refer to reader about applications and properties of the variational-like inequalities and generalized convex fuzzy mappings, see $[1,3,4,6,16,17,21,22,23$, $24,25]$, and the references therein

Motivated and inspired by the ongoing research work and by the importance of the idea of invexity and preinvexity of mappings. In Section 2, we review some basic definitions, preliminary notations and results. The main results are considered and discussed in Section 3. We have characterized preinvex fuzzy mappings in terms of $\alpha$-preinvex fuzzy mappings. In Section 3.1, the notions of $\alpha$-preinvex, quasi $\alpha$-preinvex and $\log \alpha$-preinvex fuzzy mappings are introduced and some properties are investigated. In Section 3.2, we introduce several new concepts of $\xi$-invex fuzzy mappings and $\alpha \xi$-monotonicities fuzzy operators and then discuss their relation. In Section 4 , we have shown that the minimum of $\alpha$-preinvex fuzzy mappings can be distinguished by fuzzy $\alpha$-variational like inequalities which is itself an interesting outcome of our main results.

\section{Preliminaries}

A fuzzy set on $\mathbb{R}$ is a mapping $\psi: \mathbb{R} \rightarrow[0,1]$, for each fuzzy set and $\tilde{\gamma} \in(0,1]$, then $\tilde{\gamma}$-level sets of $\psi$ is denoted and defined as follows: $\psi_{\widetilde{\gamma}}=\{u \in \mathbb{R} \mid \psi(u) \geq \tilde{\gamma}\}$. If $\tilde{\gamma}=0$, then $\operatorname{supp}(\psi)=\{u \in \mathbb{R} \mid \psi(u)>0\}$ is called support of $\psi$. By $[\psi]^{0}$ we define the closure of $\operatorname{supp}(\psi)$.

Definition 1. A fuzzy set is said to be fuzzy number with the following properties:

(a) $\psi$ is normal, i.e., there exists $u \in \mathbb{R}$ such that $\psi(u)=1$;

(b) $\psi((1-\tau) u+\tau y) \geq \min (\psi(u), \psi(y))$ for all $u, y \in \mathbb{R}, \tau \in[0,1]$;

(c) $\sup (\psi)$ is compact.

$\mathbb{F}_{0}$ denotes the set of all fuzzy numbers. For fuzzy number, it is convenient to distinguish followings $\tilde{\gamma}$-levels, 


$$
\psi_{\tilde{\gamma}}=\{u \in \mathbb{R} \mid \psi(u) \geq \tilde{\gamma}\},
$$

from these definitions, we have

$$
\psi_{\widetilde{\gamma}}=\left[\psi_{*}(\tilde{\gamma}), \psi^{*}(\tilde{\gamma})\right]
$$

where

$$
\psi_{*}(\tilde{\gamma})=\inf \{u \in \mathbb{R} \mid \psi(u) \geq \tilde{\gamma}\}, \quad \psi^{*}(\tilde{\gamma})=\sup \{u \in \mathbb{R} \mid \psi(u) \geq \tilde{\gamma}\} .
$$

Since each $r \in \mathbb{R}$ is also a fuzzy number, defined as

$$
\tilde{r}(u)= \begin{cases}1 & \text { if } u=r \\ 0 & \text { if } u \neq r\end{cases}
$$

Now we discuss some properties of fuzzy numbers under addition and scaler multiplication, if $\psi_{1}, \phi_{1} \in \mathbb{F}_{0}$ and $\rho \in \mathbb{R}$, then $\psi_{1} \widetilde{+} \phi_{1}$ and $\rho \psi_{1}$ define as

$$
\begin{gathered}
\left(\psi_{1} \widetilde{+} \phi_{1}\right)(w)=\sup _{u+y=w} \min \left\{\left(\psi_{1}\right)(w),\left(\phi_{1}\right)(w)\right\}, \\
\left(\rho \psi_{1}\right)(u)= \begin{cases}\psi_{1}\left(\rho^{-1} u\right), & \text { if } \rho \neq 0 \\
0, & \text { if } \rho=0 .\end{cases}
\end{gathered}
$$

It is also well known that for any $\psi_{1}, \phi_{1} \in \mathbb{F}_{0}$ and $\rho \in \mathbb{R}$

$$
\begin{array}{cc}
\left(\psi_{1} \tilde{+} \phi_{1}\right)^{*}(\tilde{\gamma})=\psi_{1}{ }^{*}(\tilde{\gamma})+\phi_{1}{ }^{*}(\tilde{\gamma}), & \left(\psi_{1} \widetilde{+} \phi_{1}\right)_{*}(\tilde{\gamma})=\psi_{1_{*}}(\tilde{\gamma})+\phi_{1_{*}}(\tilde{\gamma}), \\
\left(\rho \psi_{1}\right)_{*}(\tilde{\gamma})=\left\{\begin{array}{l}
\rho \psi_{1_{*}}(\tilde{\gamma}) \text { if } \rho \geq 0 \\
\rho \psi_{1}{ }^{*}(\tilde{\gamma}) \text { if } \rho<0
\end{array}, \quad\left(\rho \psi_{1}\right)^{*}(\tilde{\gamma})=\left\{\begin{array}{l}
\rho \psi_{1}{ }^{*}(\tilde{\gamma}) \text { if } \rho \geq 0 \\
\rho \psi_{1_{*}}(\tilde{\gamma}) \text { if } \rho<0
\end{array}\right.\right.
\end{array}
$$

for each $\tilde{\gamma} \in[0,1]$.

From these definitions, we have

$$
\begin{aligned}
\psi_{1} \widetilde{+} \phi_{1} & =\left\{\left(\psi_{1_{*}}(\tilde{\gamma})+\phi_{1_{*}}(\tilde{\gamma}), \psi_{1}{ }^{*}(\tilde{\gamma})+\phi_{1}{ }^{*}(\tilde{\gamma}), \tilde{\gamma}\right): \tilde{\gamma} \in[0,1]\right\}, \\
\rho \psi_{1} & =\left\{\left(\rho \psi_{1_{*}}(\tilde{\gamma}), \rho \psi_{1}{ }^{*}(\tilde{\gamma}), \tilde{\gamma}\right): \tilde{\gamma} \in[0,1]\right\} .
\end{aligned}
$$

It should be noted that for $\psi_{1} \in \mathbb{F}_{0}, r \psi_{1}$ is not a fuzzy number for $r<0$. The family of parametric representation of fuzzy numbers of $\mathbb{F}_{0}$ and the parametric representation of their negative scaler multiplications form subsets of vector spaces

$$
\begin{gathered}
\Omega=\left\{\left\{\left(\psi_{*}(\tilde{\gamma}), \psi^{*}(\tilde{\gamma}), \tilde{\gamma}\right): \tilde{\gamma} \in[0,1]\right\}: \psi_{*}:[0,1] \rightarrow \mathbb{R} \text { and } \psi^{*}:[0,1]\right. \\
\rightarrow \mathbb{R} \text { are bounded functions }\}
\end{gathered}
$$

with addition and multiplication defined level wise. 
Remark 1. Obviously, $\mathbb{F}_{0}$ is closed under addition and nonnegative scaler multiplication. Furthermore, for each scaler number $r \in \mathbb{R}$,

$$
\psi_{1} \widetilde{+} r=\left[\psi_{*}(\tilde{\gamma})+r, \psi^{*}(\tilde{\gamma})+r\right] .
$$

Definition 2. For any $\psi_{1}, \phi_{1} \in \mathbb{F}_{0}$, we say that $\psi_{1} \leq \phi_{1}$ if for all $\tilde{\gamma} \in(0,1]$, $\psi_{1}{ }^{*}(\tilde{\gamma}) \leq \phi_{1}{ }^{*}(\tilde{\gamma})$, and $\psi_{1_{*}}(\tilde{\gamma}) \leq \phi_{1_{*}}(\tilde{\gamma})$. If $\psi_{1} \leq \phi_{1}$, then there exist $\tilde{\gamma} \in(0,1]$ such that $\psi_{1}{ }^{*}(\tilde{\gamma})<\phi_{1}{ }^{*}(\tilde{\gamma})$ or $\psi_{1_{*}}(\tilde{\gamma}) \leq \phi_{1_{*}}(\tilde{\gamma})$. We say comparable if for any $\psi_{1}, \phi_{1} \in \mathbb{F}_{0}$, we have $\psi_{1} \preceq \phi_{1}$ or $\psi_{1} \geq \phi_{1}$ otherwise they are non-comparable. Some time we may write $\psi_{1} \preceq \phi_{1}$ instead of $\phi_{1} \geq \psi_{1}$ and note that, we may say that $\mathbb{F}_{0}$ is a partial ordered set under the relation $\preceq$.

If $\psi_{1}, \phi_{1} \in \mathbb{F}_{0}$, there exist $\omega_{1} \in \mathbb{F}_{0}$ such that $\psi_{1}=\phi_{1} \widetilde{+} \omega_{1}$, then by this result we have existence of Hukuhara difference of $\psi_{1}$ and $\phi_{1}$, and we say that $\omega_{1}$ is the H-difference of $\psi_{1}$ and $\phi_{1}$, and denoted by $\psi_{1} \simeq \phi_{1}$, see [21]. If $\mathrm{H}$-difference exists, then

$$
\begin{gathered}
\left(\omega_{1}\right)^{*}(\tilde{\gamma})=\left(\psi_{1} \simeq \phi_{1}\right)^{*}(\tilde{\gamma})=\psi_{1}{ }^{*}(\tilde{\gamma})-\phi_{1}{ }^{*}(\tilde{\gamma}), \\
\left(\omega_{1}\right)_{*}=\left(\psi_{1} \simeq \phi_{1}\right)_{*}(\tilde{\gamma})=\psi_{1 *}(\tilde{\gamma})-\phi_{1_{*}}(\tilde{\gamma}) .
\end{gathered}
$$

Definition 3. A mapping $\tilde{F}: K \rightarrow \mathbb{F}_{0}$ is called fuzzy mapping. For each $\tilde{\gamma} \in[0,1]$, associated to $\tilde{F}$, we define the family of interval valued functions $\tilde{F}_{\widetilde{\gamma}}: K \rightarrow \mathrm{K}_{C}$ defined by $\tilde{F}_{\widetilde{\gamma}}(u)=[\tilde{F}(u)]^{\widetilde{\gamma}}$ and denoted by $[\tilde{F}(u)]^{\widetilde{\gamma}}=\left[\tilde{F}_{*}(u, \tilde{\gamma}), \tilde{F}^{*}(u, \tilde{\gamma})\right]$. Now, for any $\tilde{\gamma} \in[0,1]$, the end point functions $\tilde{F}_{*}(u, \tilde{\gamma}), \tilde{F}^{*}(u, \tilde{\gamma}): K \rightarrow \mathbb{R}$ are called lower and upper functions, respectively.

Definition 4 (see [1]). Let $I=(p, q)$ and $u \in(p, q)$. Then fuzzy mapping $\tilde{F}:(p, q) \rightarrow \mathbb{F}_{0}$ is said to be a strongly generalized differentiable (G-differentiable) at $u$ if there exists an element $\tilde{F}^{\prime}(u) \in \mathbb{F}_{0}$ such that for all $0<t$, sufficiently small, there exist $\tilde{F}(u+t) \simeq \tilde{F}(u), \tilde{F}(u) \simeq \tilde{F}(u-t)$ and the limits (in the metric $D$ )

$$
\lim _{t \rightarrow 0^{+}} \frac{\tilde{F}(u+t) \simeq \tilde{F}(u)}{t}=\lim _{t \rightarrow 0^{+}} \frac{\tilde{F}(u) \simeq \tilde{F}(u-t)}{t}=\tilde{F}^{\prime}(u),
$$

or

$$
\lim _{t \rightarrow 0^{+}} \frac{\tilde{F}(u) \simeq \tilde{F}(u+t)}{-t}=\lim _{t \rightarrow 0^{+}} \frac{\tilde{F}(u-t) \simeq \tilde{F}(u)}{-t}=\tilde{F}^{\prime}(u),
$$

or

$$
\lim _{t \rightarrow 0^{+}} \frac{\tilde{F}(u+t) \simeq \tilde{F}(u)}{t}=\lim _{t \rightarrow 0^{+}} \frac{\tilde{F}(u-t) \simeq \tilde{F}(u)}{-t}=\tilde{F}^{\prime}(u)
$$


or

$$
\lim _{t \rightarrow 0^{+}} \frac{\tilde{F}(u) \simeq \tilde{F}(u+t)}{-t}=\lim _{t \rightarrow 0^{+}} \frac{\tilde{F}(u) \simeq \tilde{F}(u-t)}{t}=\tilde{F}^{\prime}(u),
$$

where the limits are taken in the metric space $(E, D)$, for $\psi_{1}, \psi_{2} \in \mathbb{F}_{0}$

$$
D\left(\psi_{1}, \psi_{2}\right)=\sup _{0 \leq \widetilde{\gamma} \leq 1} H\left(\psi_{1 \widetilde{\gamma}}, \psi_{2 \widetilde{\gamma}}\right)
$$

and $H$ denote the well-known Hausdorff metric on space of intervals $\mathrm{K}_{C}$.

Definition 5 (see [4]). A fuzzy mapping $\tilde{F}: K \rightarrow \mathbb{F}_{0}$ is said to be convex on the convex set $K$ if

$$
\tilde{F}((1-\tau) u+\tau y) \preceq(1-\tau) \tilde{F}(u) \widetilde{+} \tau \tilde{F}(y) \forall u, y \in K, \tau \in[0,1] .
$$

Strictly convex fuzzy mapping if strict inequality holds for $\tilde{F}(u) \neq \tilde{F}(y) . \tilde{F}: K \rightarrow \mathbb{F}_{0}$ is said to be concave fuzzy mapping if $-\tilde{F}$ is convex on $K$. Strictly concave fuzzy mapping if strict inequality holds for $\tilde{F}(u) \neq \tilde{F}(y)$.

Definition 6 (see [4]). A fuzzy mapping $\tilde{F}: K \rightarrow \mathbb{F}_{0}$ is said to be quasi-convex on the convex set $K$ if

$$
\tilde{F}((1-\tau) u+\tau y) \leq \max (\tilde{F}(u), \tilde{F}(y)) \forall u, y \in K, \tau \in[0,1] .
$$

Definition 7 (see [2]). The set $K$ in $\mathbb{R}$ is said to be invex set with respect to (with respect to) arbitrary bi-function $\xi(.,$.$) , if$

$$
u+\tau \xi(y, u) \in K, \forall u, y \in K, \tau \in[0,1] .
$$

The invex set $K$ is also called $\xi$-connected set. Note that, convex set $\xi(y, u)=y-u$ is called an invex set in classical sence, but the converse is not valid.

Definition 8 (see [22]). A fuzzy mapping $\tilde{F}: K \rightarrow \mathbb{F}_{0}$ is said to be preinvex on invex set $K$ with respect to bi-function $\xi$ if

$$
\tilde{F}(u+\tau \xi(y, u)) \preceq(1-\tau) \tilde{F}(u) \widetilde{+} \tau \tilde{F}(y) \forall u, y \in K, \tau \in[0,1],
$$

where $\xi: K \times K \rightarrow \mathbb{R}$.

Strictly preinvex fuzzy mapping if strict inequality holds for $\tilde{F}(u) \neq \tilde{F}(y) . \tilde{F}: K \rightarrow \mathbb{F}_{0}$ is said to be preconcave fuzzy mapping if $-\tilde{F}$ is preinvex on $K$. Strictly preconcave fuzzy mapping if strict inequality holds for $\tilde{F}(u) \neq \tilde{F}(y)$. 
Definition 9 (see [22]). The fuzzy mapping $\tilde{F}: K \rightarrow \mathbb{F}_{0}$ is said to be quasi-preinvex on invex set $K$ with respect to $\xi$ if

$$
\tilde{F}(u+\tau \xi(y, u)) \leq \max (\tilde{F}(u), \tilde{F}(y)) \forall u, y \in K, \tau \in[0,1] .
$$

Definition 10 (see [14]). A fuzzy mapping $\tilde{F}: K \rightarrow \mathbb{F}_{0}$ is said to be log-preinvex on invex set $K$ with respect to bi-function $\xi$ if there exist a positive number $\omega$ such that

$$
\tilde{F}(u+\tau \xi(y, u)) \leq(\tilde{F}(u))^{1-\tau}(\tilde{F}(y))^{\tau} \forall u, y \in K, \tau \in[0,1],
$$

where $\tilde{F}()>.\tilde{0}$.

Definition 11 (see [10]). The set $K_{\alpha}$ is said to be $\alpha$-invex set with respect to arbitrary bifunctions $\xi(.,$.$) and \alpha(.,$.$) , if$

$$
u+\tau \alpha(y, u) \xi(y, u) \in K_{\alpha}, \forall u, y \in K_{\alpha}, \tau \in[0,1] .
$$

The $\alpha$-invex set $K_{\alpha}$ is also called $\alpha \xi$-connected set. Note that, convex set with $\alpha(y, u)=1$ and $\xi(y, u)=y-u$ is called an invex set in classical sense, but the converse is not valid. For example, see [12], the following set $K_{\alpha}=[-7,-2] \cup[2,10]$ is an invex set with respect to non-trivial bi-function $\xi: \mathbb{R} \times \mathbb{R} \rightarrow \mathbb{R}$ and $\alpha(y, u)=1$, given as

$$
\begin{array}{lc}
\tilde{\xi}(y, u)=y-u, & y \geq 0, u \geq 0 \\
\tilde{\xi}(y, u)=y-u, & 0 \geq y, 0 \geq u \\
\tilde{\xi}(y, u)=-7-u, & y \geq 0 \geq u \\
\tilde{\xi}(y, u)=2-u, & u \geq 0 \geq y .
\end{array}
$$

\section{Remark 2.}

(i) If $\alpha(y, u)=1$, then the set $K_{\alpha}$ is an invex set.

(ii) If $0<\alpha(y, u)<1$ and $\xi(y, u)=y-u$, then the set $K_{\alpha}$ is called starshaped.

(iii) If $\alpha(y, u)=1$ and $\xi(y, u)=y-u$, then the set $K_{\alpha}$ is called convex.

\section{Main Results}

Let $K_{\alpha}$ be a nonempty $\alpha$-invex subset of $\mathbb{R}$ with respect to $\xi, \alpha$. Let $\tilde{F}: K_{\alpha} \rightarrow \mathbb{F}_{0}$ be continuous mapping and $\xi: K_{\alpha} \times K_{\alpha} \rightarrow \mathbb{R}$ be an arbitrary continuous bi-function. Let 
$\alpha: K_{\alpha} \times K_{\alpha} \rightarrow \mathbb{R} \backslash 0$ be a bi-function. We denote $\|$.$\| and \langle.,$.$\rangle the norm and inner product,$ respectively.

\section{1. $\alpha$-preinvex fuzzy mappings}

Definition 12. Let $K_{\alpha}$ be a $\alpha$-invex set with respect to $\xi, \alpha$. Then fuzzy mapping $\tilde{F}: K_{\alpha} \rightarrow \mathbb{F}_{0}$ is said to be $\alpha$-preinvex with respect to bi-functions $\xi(.,$.$) and \alpha(.,$.$) if$

$$
\tilde{F}(u+\tau \alpha(y, u) \xi(y, u)) \leq(1-\tau) \tilde{F}(u) \widetilde{+} \tau \tilde{F}(y) \forall u, y \in K_{\alpha}, \tau \in[0,1] .
$$

Strictly $\alpha$-preinvex fuzzy mapping if strict inequality holds for $\tilde{F}(u) \neq \tilde{F}(y)$ and $\tilde{F}: K_{\alpha} \rightarrow \mathbb{F}_{0}$ is said to be $\alpha$-preconcave fuzzy mapping if $-\tilde{F}$ is $\alpha$-preinvex on $K_{\alpha}$. Strictly $\alpha$-preconcave fuzzy mapping if strict inequality holds for $\tilde{F}(u) \neq \tilde{F}(y)$. Note that every convex fuzzy mapping with $\alpha(y, u)=1$ and $\xi(y, u)=y-u$ is called preinvex fuzzy mapping but converse does not hold, see example 1 .

Now we discuss some special cases of $\alpha$-preinvex fuzzy mappings:

(i) If $\alpha(y, u)=1$, then $\alpha$-preinvex fuzzy mapping becomes preinvex fuzzy mapping, that is

$$
\tilde{F}(u+\tau \xi(y, u)) \leq(1-\tau) \tilde{F}(u) \widetilde{+} \tau \tilde{F}(y) \forall u, y \in K_{\alpha}, \tau \in[0,1] .
$$

(ii) If $0<\alpha(y, u)<1$, then $\alpha$-preinvex fuzzy mapping is called star-shaped $\alpha$-preinvex fuzzy mapping.

(iii) If $\alpha(y, u)=1$ and $\xi(y, u)=y-u$, then $\alpha$-preinvex fuzzy mapping becomes convex fuzzy mapping, that is

$$
\tilde{F}(u+\tau(y-u)) \leq(1-\tau) \tilde{F}(u) \widetilde{+} \tau \tilde{F}(y) \forall u, y \in K_{\alpha}, \tau \in[0,1] .
$$

(iv) If $0<\alpha(y, u)<1$ and $\tau=\frac{1}{2}$, then (3.1) becomes

$$
\tilde{F}\left(\frac{2 u+\alpha(y, u) \xi(y, u)}{2}\right) \leq \frac{\tilde{F}(u) \tilde{f} \tilde{F}(y)}{2} \forall u, y \in K_{\alpha} .
$$

The mapping $\tilde{F}$ is called the star-shaped $J$ - $\alpha$-preinvex fuzzy mapping.

(v) If $\tau=\frac{1}{2}$, then (3.1) becomes

$$
\tilde{F}\left(\frac{2 u+\alpha(y, u) \xi(y, u)}{2}\right) \preceq \frac{\tilde{F}(u) \tilde{+} \tilde{F}(y)}{2} \forall u, y \in K_{\alpha} .
$$

The fuzzy mapping $\tilde{F}$ is called the $J$ - $\alpha$-preinvex. For $\alpha(y, u)=1$, (3.2) reduces to 


$$
\tilde{F}\left(\frac{2 u+\xi(y, u)}{2}\right) \leq \frac{\tilde{F}(u) \widetilde{+} \tilde{F}(y)}{2} \forall u, y \in K_{\alpha},
$$

then fuzzy mapping $\tilde{F}$ is called the $J$-preinvex.

We also define the affine $J$ - $\alpha$-preinvex mapping.

Definition 13. A fuzzy mapping $\tilde{F}: K \rightarrow \mathbb{F}_{0}$ is said to be affine $\alpha$-preinvex on the $\alpha$-invex set $K_{\alpha}$ with respect to $\xi, \alpha$, if

$$
\tilde{F}(u+\tau \alpha(y, u) \xi(y, u))=(1-\tau) \tilde{F}(u) \tilde{+} \tau \tilde{F}(y), \forall u, y \in K_{\alpha}, \tau \in[0,1] .
$$

Note that, if a fuzzy mapping is both $\alpha$-preinvex and $\alpha$-preconcave, then it is a affine $\alpha$-preinvex fuzzy mapping with respect to $\xi, \alpha$.

If $\tau=\frac{1}{2}$, then fuzzy mapping $\tilde{F}$ is called a quadratic equation with respect to $\xi, \alpha$ such that

$$
\tilde{F}\left(\frac{2 u+\alpha(y, u) \xi(y, u)}{2}\right)=\frac{\tilde{F}(u) \widetilde{+} \tilde{F}(y)}{2}, \forall u, y \in K_{\alpha} .
$$

This fuzzy mapping is also called affine $J$ - $\alpha$-preinvex.

Proposition 1. If $\tilde{F}_{j}: K_{\alpha} \rightarrow \mathbb{F}_{0}$ be a $\alpha$-preinvex with respect to $\xi, \alpha$ and $\rho_{j} \geq 0$, $j=1,2,3, \ldots, m$, then $\sum_{j=1}^{m} \rho_{j} \tilde{F}_{j}(u)$ is $\alpha$-preinvex.

Proof. Let $\tilde{F}_{j}: K_{\alpha} \rightarrow \mathbb{F}_{0}$ be $\alpha$-preinvex such that

$$
\tilde{F}_{j}(u+\tau \alpha(y, u) \xi(y, u)) \leq(1-\tau) \tilde{F}_{j}(u) \widetilde{+} \tau \tilde{F}_{j}(y), j=1,2,3, \ldots, m,
$$

now by the convex combination,

$$
\begin{aligned}
\tau \sum_{j=1}^{m} \rho_{j} \tilde{F}_{j}(u) \widetilde{+}(1-\tau) \sum_{j=1}^{m} \rho_{j} \tilde{F}_{j}(y) & =\sum_{j=1}^{m} \rho_{j}\left\{(1-\tau) \tilde{F}_{j}(u) \widetilde{+}_{\tau} \tilde{F}_{j}(y)\right\}, \\
& \geqslant \sum_{j=1}^{m} \rho_{j} \tilde{F}_{j}(u+\tau \alpha(y, u) \xi(y, u)),
\end{aligned}
$$

which implies that the $\sum_{j=1}^{m} \rho_{j} \tilde{F}_{j}(u)$ is $\alpha$-preinvex on $K_{\alpha}$ with respect to $\xi, \alpha$.

Theorem 1. Let $K_{\alpha}$ be a $\alpha$-invex set with respect to $\xi, \alpha$, and let $\tilde{F}: K_{\alpha} \rightarrow \mathbb{F}_{0}$ be a fuzzy mapping parametrized by

$$
\tilde{F}(u)=\left\{\left(\tilde{F}_{*}(u, \tilde{\gamma}), \tilde{F}^{*}(u, \tilde{\gamma}), \tilde{\gamma}\right): \tilde{\gamma} \in[0,1]\right\}, \forall u \in K_{\alpha} .
$$

Then $\tilde{F}$ is $\alpha$-preinvex on $K_{\alpha}$ with respect to $\xi, \alpha$ if and only if, for all $\tilde{\gamma} \in[0,1]$,

$$
\tilde{F}_{*}(u, \tilde{\gamma}) \text { and } \tilde{F}^{*}(u, \tilde{\gamma}) \text { are } \alpha \text {-preinvex with respect to } \xi, \alpha \text {. }
$$


Proof. Assume that for each $\tilde{\gamma} \in[0,1], \tilde{F}_{*}(u, \tilde{\gamma})$ and $\tilde{F}^{*}(u, \tilde{\gamma})$ are $\alpha$-preinvex with respect to $\xi, \alpha$ on $K_{\alpha}$. Then from (3.4), we have

$$
\tilde{F}_{*}(u+\tau \alpha(y, u) \xi(y, u), \tilde{\gamma}) \leq(1-\tau) \tilde{F}_{*}(u, \tilde{\gamma})+\tau \tilde{F}_{*}(y, \tilde{\gamma}) \forall u, y \in K_{\alpha}, \tau \in[0,1],
$$
and

$$
\tilde{F}^{*}(u+\tau \alpha(y, u) \xi(y, u), \tilde{\gamma}) \leq(1-\tau) \tilde{F}^{*}(u, \tilde{\gamma})+\tau \tilde{F}^{*}(y, \tilde{\gamma}) \forall u, y \in K_{\alpha}, \tau \in[0,1] .
$$

Then by (3.3), (2.1) and (2.2), we obtain

$$
\begin{aligned}
& \tilde{F}(u+\tau \alpha(y, u) \xi(y, u)) \\
= & \left\{\left(\tilde{F}_{*}(u+\tau \alpha(y, u) \xi(y, u), \tilde{\gamma}), \tilde{F}^{*}(u+\tau \alpha(y, u) \xi(y, u), \tilde{\gamma}), \tilde{\gamma}\right): \tilde{\gamma} \in[0,1]\right\}, \\
\leq & \left\{\left((1-\tau) \tilde{F}_{*}(u, \tilde{\gamma}),(1-\tau) \tilde{F}^{*}(u, \tilde{\gamma}), \tilde{\gamma}\right): \tilde{\gamma} \in[0,1]\right\} \\
& \widetilde{F}\left\{\left(\tau \tilde{F}_{*}(y, \tilde{\gamma}), \tau \tilde{F}^{*}(y, \tilde{\gamma}), \tilde{\gamma}\right): \tilde{\gamma} \in[0,1]\right\}, \\
= & (1-\tau) \tilde{F}(u) \widetilde{+} \tau \tilde{F}(y),
\end{aligned}
$$

for all $u, y \in K_{\alpha}$ and $\tau \in[0,1]$. Hence $\tilde{F}$ is $\alpha$-preinvex fuzzy mapping on $K_{\alpha}$.

Conversely, let $\tilde{F}$ is $\alpha$-preinvex mapping on $K_{\alpha}$. Then for all $u, y \in K_{\alpha}$ and $\tau \in[0,1]$, we have $\tilde{F}(u+\tau \alpha(y, u) \xi(y, u)) \preceq(1-\tau) \tilde{F}(u) \tilde{+} \tau \tilde{F}(y)$. From (3.3), for all $u, y \in$ $K_{\alpha}, \tau \in[0,1]$ we have

$$
\begin{aligned}
& \tilde{F}(u+\tau \alpha(y, u) \xi(y, u)) \\
= & \left\{\left(\tilde{F}_{*}(u+\tau \alpha(y, u) \xi(y, u), \tilde{\gamma}), \tilde{F}^{*}(u+\tau \alpha(y, u) \xi(y, u), \tilde{\gamma}), \tilde{\gamma}\right): \tilde{\gamma} \in[0,1]\right\} .
\end{aligned}
$$

From (3.3), (2.1) and (2.2), we obtain

$$
\begin{aligned}
(1-\tau) \tilde{F}(u) \tilde{+} \tau \tilde{F}(y)= & \left\{\left((1-\tau) \tilde{F}_{*}(u, \tilde{\gamma}),(1-\tau) \tilde{F}^{*}(u, \tilde{\gamma}), \tilde{\gamma}\right): \tilde{\gamma} \in[0,1]\right\} \\
& \widetilde{\mp}\left\{\left(\tau \tilde{F}_{*}(y, \tilde{\gamma}), \tau \tilde{F}^{*}(y, \tilde{\gamma}), \tilde{\gamma}\right): \tilde{\gamma} \in[0,1]\right\},
\end{aligned}
$$

for all $u, y \in K_{\alpha}$ and $\tau \in[0,1]$. Then by $\alpha$-preinvexity of $\tilde{F}$, we have for all $u, y \in K_{\alpha}$ and $\tau \in[0,1]$ such that

$$
\tilde{F}_{*}(u+\tau \alpha(y, u) \xi(y, u), \tilde{\gamma}) \leq(1-\tau) \tilde{F}_{*}(u, \tilde{\gamma})+\tau \tilde{F}_{*}(y, \tilde{\gamma}),
$$

and

$$
\tilde{F}^{*}(u+\tau \alpha(y, u) \xi(y, u), \tilde{\gamma}) \leq(1-\tau) \tilde{F}^{*}(u, \tilde{\gamma})+\tau \tilde{F}^{*}(y, \tilde{\gamma}),
$$

for each $\tilde{\gamma} \in[0,1]$. Hence, the result follows. 
Example 1. We consider the fuzzy mappings $\tilde{F}:(-1,1) \rightarrow \mathbb{F}_{0}$ defined by,

$$
\tilde{F}(u)(\sigma)= \begin{cases}\frac{\sigma-u^{2}}{1-u^{2}}, & \sigma \in\left[u^{2}, 1\right) \\ 0, & \text { otherwise. }\end{cases}
$$

Then, for each $\tilde{\gamma} \in[0,1]$, we have $\tilde{F}_{\widetilde{\gamma}}(u)=\left[\tilde{\gamma}+(1-\tilde{\gamma}) u^{2}, 1\right]$. Since end point functions $\tilde{F}_{*}(\tilde{\gamma}), \tilde{F}^{*}(\tilde{\gamma})$ are $\alpha$-preinvex for each $\tilde{\gamma} \in[0,1]$, then $\tilde{F}$ is $\alpha$-preinvex fuzzy mapping with respect to bi-functions

$$
\xi(y, u)= \begin{cases}\frac{y^{2}-u^{2}}{2 u}, & y^{2}-u^{2}<0 \\ 0, & \text { otherwise }\end{cases}
$$

and $\alpha(y, u)=1$.

Definition 14. A set $K_{\alpha} \subseteq \mathbb{R}^{2}$ is said to an $\alpha$-invex set with respect to $\xi, \alpha$ if

$$
\begin{gathered}
\left(u+\tau \alpha(y, u) \xi(y, u),(1-\tau) \tilde{\gamma}_{1} \tilde{\tau} \tau \tilde{\gamma}_{2}\right) \in K_{\alpha}, \\
\left(u, \tilde{\gamma}_{1}\right),\left(y, \tilde{\gamma}_{2}\right) \in K_{\alpha}, u, y \in \mathbb{R}, \tau \in[0,1] .
\end{gathered}
$$

The epigraph of $\alpha$-preinvex fuzzy mapping $\tilde{F}: K_{\alpha} \rightarrow \mathbb{F}_{0}$ can be given as

$$
G(\tilde{F})=\left\{(u, \tilde{\gamma}): u \in K_{\alpha}, \tilde{\gamma} \in \mathbb{F}_{0}, \tilde{F}(u) \preceq \tilde{\gamma}\right\} .
$$

We now give a characterization of $\alpha$-preinvex fuzzy mappings in terms of its epigraph $G(\tilde{F})$.

Theorem 2. A fuzzy mapping $\tilde{F}: K_{\alpha} \rightarrow \mathbb{F}_{0}$ is a $\alpha$-preinvex with respect to $\xi, \alpha$ if and only if, its epigraph is an $\tilde{\alpha}$-invex set with respect to $\tilde{\xi}, \tilde{\alpha}$, where $\tilde{\xi}: G(\tilde{F}) \times G(\tilde{F}) \rightarrow \mathbb{R} \times$ $\Omega$ and $\tilde{\alpha}: G(\tilde{F}) \times G(\tilde{F}) \rightarrow \mathbb{R} \backslash 0 \times \Omega$ with

$$
\tilde{\alpha}\left(\left(y, \tilde{\gamma}_{2}\right),\left(u, \tilde{\gamma}_{1}\right)\right) \tilde{\xi}\left(\left(y, \tilde{\gamma}_{2}\right),\left(u, \tilde{\gamma}_{1}\right)\right)=\left(\alpha(y, u) \xi(y, u), \tilde{\gamma}_{2} \widetilde{+}(-1) \tilde{\gamma}_{1}\right),
$$

$\operatorname{for}\left(u, \tilde{\gamma}_{1}\right),\left(y, \tilde{\gamma}_{2}\right) \in G(\tilde{F})$.

Proof. Let $\left(u, \tilde{\gamma}_{1}\right),\left(y, \tilde{\gamma}_{2}\right) \in G(\tilde{F})$. Then $\tilde{F}(u) \leq \tilde{\gamma}_{1}$ and $\tilde{F}(y) \leq \tilde{\gamma}_{2}$. Since fuzzy mapping $\tilde{F}: K_{\alpha} \rightarrow \mathbb{F}_{0}$ is $\alpha$-preinvex with respect to $\xi, \alpha$ so for all $u, y \in K_{\alpha}$ and $\tau \in[0,1]$, we have

$$
\tilde{F}(u+\tau \alpha(y, u) \xi(y, u)) \preceq(1-\tau) \tilde{F}(u) \widetilde{+} \tau \tilde{F}(y) \preceq(1-\tau) \tilde{\gamma}_{1} \widetilde{+} \tilde{\gamma}_{2},
$$


from which it follows that

$$
\left(u+\tau \alpha(y, u) \xi(y, u),(1-\tau) \tilde{\gamma}_{1} \widetilde{+} \tau \tilde{\gamma}_{2}\right) \in G(\tilde{F}),
$$

which implies that

$$
\begin{aligned}
\left(u+\tau \alpha(y, u) \xi(y, u),(1-\tau) \tilde{\gamma}_{1} \tilde{+} \tau \tilde{\gamma}_{2}\right) & =\left(u, \tilde{\gamma}_{1}\right)+\tau\left(\alpha(y, u) \xi(y, u), \tilde{\gamma}_{2} \tilde{+}(-1) \tilde{\gamma}_{1}\right) \\
& =\left(u, \tilde{\gamma}_{1}\right)+\tau \tilde{\alpha}\left(\left(y, \tilde{\gamma}_{2}\right),\left(u, \tilde{\gamma}_{1}\right)\right) \tilde{\xi}\left(\left(y, \tilde{\gamma}_{2}\right),\left(u, \tilde{\gamma}_{1}\right)\right) \\
& \in G(\tilde{F}) .
\end{aligned}
$$

Hence, $G(\tilde{F})$ is an $\tilde{\alpha}$-invex set with respect to $\tilde{\xi}: G(\tilde{F}) \times G(\tilde{F}) \rightarrow \mathbb{R} \times \Omega, \tilde{\alpha}: G(\tilde{F}) \times$ $G(\tilde{F}) \rightarrow \mathbb{R} \backslash 0 \times \Omega$ with $\tilde{\alpha}\left(\left(y, \tilde{\gamma}_{2}\right),\left(u, \tilde{\gamma}_{1}\right)\right) \tilde{\xi}\left(\left(y, \tilde{\gamma}_{2}\right),\left(u, \tilde{\gamma}_{1}\right)\right)=\left(\alpha(y, u) \xi(y, u), \tilde{\gamma}_{2} \tilde{+}(-1) \tilde{\gamma}_{1}\right)$ for $\left(u, \tilde{\gamma}_{1}\right),\left(y, \tilde{\gamma}_{2}\right) \in G(\tilde{F})$.

Conversely, $G(\tilde{F})$ is an $\tilde{\alpha}$-invex set with respect to $\tilde{\xi}, \tilde{\alpha}: G(\tilde{F}) \times G(\tilde{F}) \rightarrow \mathbb{R} \times \Omega$, with $\tilde{\alpha}\left(\left(y, \tilde{\gamma}_{2}\right),\left(u, \tilde{\gamma}_{1}\right)\right) \tilde{\xi}\left(\left(y, \tilde{\gamma}_{2}\right),\left(u, \tilde{\gamma}_{1}\right)\right)=\left(\alpha(y, u) \xi(y, u), \tilde{\gamma}_{2} \widetilde{+}(-1) \tilde{\gamma}_{1}\right)$ for $\left(y, \tilde{\gamma}_{2}\right),\left(u, \tilde{\gamma}_{1}\right) \in$ $G(\tilde{F})$. Since $(u, \tilde{F}(u)),(y, \tilde{F}(y)) \in G(\tilde{F})$, we have for $\tau \in[0,1]$

$$
(u, \tilde{F}(u))+\tau \tilde{\alpha}((y, \tilde{F}(y)),(u, \tilde{F}(u))) \tilde{\xi}((y, \tilde{F}(y)),(u, \tilde{F}(u))) \in G(\tilde{F}),
$$

since $\tilde{\alpha}\left(\left(y, \tilde{\gamma}_{2}\right),\left(u, \tilde{\gamma}_{1}\right)\right) \tilde{\xi}\left(\left(y, \tilde{\gamma}_{2}\right),\left(u, \tilde{\gamma}_{1}\right)\right)=\left(\alpha(y, u) \xi(y, u), \tilde{\gamma}_{2} \tilde{+}(-1) \tilde{\gamma}_{1}\right)$, then

$$
(u, \tilde{F}(u))+\tau(\alpha(y, u) \xi(y, u), \tilde{F}(y) \widetilde{+}(-1) \tilde{F}(u)) \in G(\tilde{F}),
$$

which implies that

$$
(u+\tau \alpha(y, u) \xi(y, u),(1-\tau) \tilde{F}(u) \tilde{+} \tau \tilde{F}(y)) \in G(\tilde{F}),
$$

so we have

$$
\tilde{F}(u+\tau \alpha(y, u) \xi(y, u)) \leq(1-\tau) \tilde{F}(u) \tilde{+} \tau \tilde{F}(y) .
$$

Hence, $\tilde{F}: K_{\alpha} \rightarrow \mathbb{F}_{0}$ is a $\alpha$-preinvex fuzzy mapping with respect to $\xi, \alpha$.

Definition 15. A fuzzy mapping $\tilde{F}: K_{\alpha} \rightarrow \mathbb{F}_{0}$ is said to be quasi $\alpha$-preinvex on $K_{\alpha}$ with respect to bi-functions $\xi, \alpha$ such that

$$
\tilde{F}(u+\tau \alpha(y, u) \xi(y, u)) \leq \max (\tilde{F}(u), \tilde{F}(y)) \forall u, y \in K_{\alpha}, \tau \in[0,1] .
$$

Similarly, A fuzzy mapping $\tilde{F}$ is said to be quasi $\alpha$-preconcave if $-\tilde{F}$ is quasi $\alpha$-preinvex on $K$. 
If $\alpha(y, u)=1$, then quasi $\alpha$-preinvex fuzzy mapping becomes quasi-preinvex fuzzy mapping in classical sense, that is

$$
\tilde{F}(u+\tau \xi(y, u)) \preceq \max (\tilde{F}(u), \tilde{F}(y)) \forall u, y \in K_{\alpha}, \tau \in[0,1] .
$$

Proposition 2. Let $\tilde{F}: K_{\alpha} \rightarrow \mathbb{F}_{0}$ be a $\alpha$-preinvex fuzzy mapping with respect to $\xi, \alpha$, such that $\tilde{F}(y) \prec \tilde{F}(u)$. Then $\tilde{F}$ is strictly quasi $\alpha$-preinvex fuzzy mapping with respect to $\xi, \alpha$.

Proof. Let $\tilde{F}(y) \prec \tilde{F}(u)$ and $\tilde{F}$ be $\alpha$-preinvex fuzzy mapping. Then, for all $u, y \in K_{\alpha}$ and $\tau \in[0,1]$ we have

$$
\tilde{F}(u+\tau \alpha(y, u) \xi(y, u)) \leq(1-\tau) \tilde{F}(u) \widetilde{+} \tau \tilde{F}(y),
$$

since $\tilde{F}(y) \prec \tilde{F}(u)$, we have

$$
\tilde{F}(u+\tau \alpha(y, u) \xi(y, u)) \prec \tilde{F}(u) .
$$

Hence, $\tilde{F}$ is strictly quasi $\alpha$-preinvex fuzzy mapping with respect to $\xi, \alpha$.

Definition 16. A fuzzy mapping $\tilde{F}: K_{\alpha} \rightarrow \mathbb{F}_{0}$ is said to be $\log \alpha$-preinvex on $K_{\alpha}$ with respect to bi-function $\xi, \alpha$ such that

$$
\tilde{F}(u+\tau \alpha(y, u) \xi(y, u)) \leq(\tilde{F}(u))^{1-\tau}(\tilde{F}(y))^{\tau} \forall u, y \in K_{\alpha}, \tau \in[0,1],
$$

where $\tilde{F}()>.\tilde{0}$.

Similarly, a fuzzy mapping $\tilde{F}$ is said to be $\log \alpha$-preconcave if $-\tilde{F}$ is $\log \alpha$-preinvex on $K_{\alpha}$.

If $\alpha(y, u)=1$, then (3.6) becomes

$$
\tilde{F}(u+\tau \xi(y, u)) \leq(\tilde{F}(u))^{1-\tau}(\tilde{F}(y))^{\tau} \forall u, y \in K_{\alpha}, \tau \in[0,1],
$$

where $\tilde{F}()>.\tilde{0}$.

The mapping $\tilde{F}$ is log-preinvex fuzzy mapping with respect to $\xi$.

From Definition 16, we have

$$
\begin{aligned}
\tilde{F}(u+\tau \alpha(y, u) \xi(y, u)) & \leq(\tilde{F}(u))^{1-\tau}(\tilde{F}(y))^{\tau} \\
& \leq(1-\tau) \tilde{F}(u) \tilde{F} \tau \tilde{F}(y) \\
& \leq \max (\tilde{F}(u), \tilde{F}(y)) .
\end{aligned}
$$


It can easily be seen that $\log \alpha$-preinvex fuzzy mapping $\Rightarrow \alpha$-preinvex fuzzy mapping $\Rightarrow$ quasi $\alpha$-preinvex fuzzy mapping.

For $\tau=1$, Definition 12 and Definition 16, reduces to:

\section{Condition A.}

$$
\tilde{F}(u+\alpha(y, u) \xi(y, u)) \leq \tilde{F}(y) \text { for all } u, y \in K_{\alpha} .
$$

Which plays an important role in studying the properties of $\alpha$-preinvex fuzzy mappings and $\alpha$-invex fuzzy mappings. If $\alpha(y, u)=1$, then Condition A reduces to the following for preinvex fuzzy mappings.

\section{Condition B.}

$$
\tilde{F}(u+\xi(y, u)) \preceq \tilde{F}(y) \text { for all } u, y \in K_{\alpha} .
$$

For the applications of Condition B, see [14, 16, 20, 23].

Definition 17. A fuzzy mapping $\tilde{F}: K_{\alpha} \rightarrow \mathbb{F}_{0}$ is said to be pseudo $\alpha$-preinvex fuzzy mapping on the $\alpha$-invex set $K_{\alpha}$ if there exist a strictly positive bi-function $b(.,$.$) such$ that $\tilde{F}(y) \prec \tilde{F}(u) \Rightarrow \tilde{F}(u+\tau \alpha(y, u) \xi(y, u)) \prec \tilde{F}(u) \widetilde{F} \tau(\tau-1) b(u, y)$, for all $u, y \in$ $K_{\alpha}, t \in[1,0]$.

Proposition 3. Let $\tilde{F}$ be a pseudo $\alpha$-preinvex fuzzy mapping on $K_{\alpha}$ such that $\tilde{F}(y) \prec \tilde{F}(u)$. Then fuzzy mapping $\tilde{F}$ is pseudo $\alpha$-preinvex.

Proof. Let $\tilde{F}(y) \prec \tilde{F}(u)$ and $\tilde{F}$ be a pseudo $\alpha$-preinvex fuzzy mapping. Then

$$
\begin{aligned}
\tilde{F}(u+\tau \xi(y, u)) & \leq(1-\tau) \tilde{F}(u) \widetilde{+} \tau \tilde{F}(y), \text { for all } u, y \in K_{\alpha}, t \in[1,0] \\
& =\tilde{F}(u) \widetilde{+} \tau(\tilde{F}(y) \simeq \tilde{F}(u)) \\
& \prec \tilde{F}(u) \widetilde{+} \tau(\tau-1)(\tilde{F}(u) \simeq \tilde{F}(y)) \\
& =\tilde{F}(u) \widetilde{+} \tau(\tau-1) b(u, y),
\end{aligned}
$$

where $b(u, y)=\tilde{F}(u) \simeq \tilde{F}(y)$. This prove that $\tilde{F}$ is pseudo $\alpha$-preinvex fuzzy mapping.

\subsection{G-differentiable $\alpha$-preinvex fuzzy mappings}

In this section, we have proposed the concepts of $\alpha$-invex fuzzy mappings and $\alpha \xi$ monotone fuzzy operators. With the support these notions, we have investigated some properties of G-differentiable $\alpha$-preinvex fuzzy mappings. 
Definition 18. A fuzzy mapping $\tilde{F}: K_{\alpha} \rightarrow \mathbb{F}_{0}$ is said to be sharply pseudo $\alpha$-preinvex on the $\alpha$-invex set $K_{\alpha}$ if

$$
\begin{array}{r}
\langle\tilde{F}(u), \alpha(y, u) \xi(y, u)\rangle \geq \tilde{0} \Rightarrow \tilde{F}(y+\tau \alpha(y, u) \xi(y, u)) \leq \tilde{F}(y), \\
\qquad \text { for all } u, y \in K_{\alpha}, t \in[1,0]
\end{array}
$$

where $\tilde{F}$, is G-differentiable of $\tilde{F}$ at $u$.

Theorem 3. Let $\tilde{F}$ be a sharply pseudo $\alpha$-preinvex fuzzy mapping on the $K_{\alpha}$. Then

$$
\simeq\left\langle\tilde{F}^{\prime}(u), \alpha(y, u) \xi(y, u)\right\rangle \geq \tilde{0}, \text { for all } u, y \in K_{\alpha} .
$$

Proof. Let $\tilde{F}$ be a sharply pseudo $\alpha$-preinvex fuzzy mapping. Then

$$
\tilde{F}(y) \geq \tilde{F}(y+\tau \alpha(y, u) \xi(y, u)) \text {, for all } u, y \in K_{\alpha}, t \in[1,0],
$$

from which we get

$$
\frac{\tilde{F}(y+\tau \alpha(y, u) \xi(y, u)) \simeq \tilde{F}(u)}{\tau} \leq \tilde{0}
$$

taking limit in the above inequality as $\tau \rightarrow \mathbf{0}$, we get

$$
\simeq\left\langle\tilde{F}^{\prime}(u), \xi(y, u)\right\rangle \geq \tilde{0}, \text { for all } u, y \in K_{\alpha} .
$$

Hence, the result follows.

We need the following assumption regarding the bi-function $\xi, \alpha$ which plays an important role in G-differentiation of the main results.

Condition C [16].

$$
\begin{gathered}
\xi(y, u+\tau \alpha(y, u) \xi(y, u))=(1-\tau) \xi(y, u), \\
\xi(u, u+\tau \alpha(y, u) \xi(y, u))=-\tau \xi(y, u), \text { for all } u, y \in K_{\alpha}, \tau \in[0,1] .
\end{gathered}
$$

Clearly for $\tau=0$, we have $\xi(u, u)=0$ for all $u \in K_{\alpha}$.

It is well known that, each G-differentiable preinvex fuzzy mapping is invex fuzzy mapping but to prove its converse we need special condition.

It can easily be seen that, if $\alpha(y, u)=1$, then Condition $\mathrm{C}$ collapse to the following condition which is due to Neogy and Mohen [13].

\section{Condition D.}

$$
\xi(y, u+\tau \xi(y, u))=(1-\tau) \xi(y, u),
$$




$$
\xi(u, u+\tau \xi(y, u))=-\tau \xi(y, u), \text { for all } u, y \in K_{\alpha}, \tau \in[0,1] .
$$

For the applications of Condition D, see [13, 14, 16, 20, 23].

Definition 19. The G-differentiable fuzzy mapping $\tilde{F}: K_{\alpha} \rightarrow \mathbb{F}_{0}$ on the $\alpha$-invex set $K_{\alpha}$ is said to be $\alpha$-invex fuzzy mapping with respect to $\xi, \alpha$ if

$$
\tilde{F}(y) \simeq \tilde{F}(u) \geq\langle\alpha(y, u) \tilde{F}(u), \xi(y, u)\rangle, \text { for all } u, y \in K_{\alpha} .
$$

Definition 20. The G-differentiable fuzzy mapping $\tilde{F}: K_{\alpha} \rightarrow \mathbb{F}_{0}$ on $K_{\alpha}$ is said to be pseudo $\alpha \xi$-invex fuzzy mapping with respect to bi-function $\xi$ if

$$
\left\langle\alpha(y, u) \tilde{F}^{\prime}(u), \xi(y, u)\right\rangle \geq \tilde{0} \Rightarrow \tilde{F}(y) \simeq \tilde{F}(u) \geq \tilde{0}, \text { for all } u, y \in K_{\alpha} .
$$

Definition 21. The G-differentiable fuzzy mapping $\tilde{F}: K_{\alpha} \rightarrow \mathbb{F}_{0}$ on $K_{\alpha}$ is said to be quasi $\alpha \xi$-invex fuzzy mapping with respect to bi-function $\xi$ if

$$
\tilde{F}(y) \preceq \tilde{F}(u) \Longrightarrow\left\langle\alpha(y, u) \tilde{F}^{\prime}(u), \xi(y, u)\right\rangle \preceq \tilde{0}, \text { for all } u, y \in K_{\alpha} .
$$

If $\xi(y, u)=-\xi(u, y)$, then definitions reduce to known ones. These definitions may play important role in fuzzy optimization problem and mathematical programming.

Theorem 4. Let $\tilde{F}: K_{\alpha} \rightarrow \mathbb{F}_{0}$ be a G-differentiable $\alpha$-preinvex fuzzy mapping with respect to $\xi, \alpha$. Let Condition $C$ hold and $\alpha(u, w)=\alpha(y, w)$ for all $u, y, w \in K_{\alpha}$. Then $\tilde{F}$ is $\alpha$-preinvex fuzzy mapping if and only if $\tilde{F}$ is $\alpha$-invex fuzzy mapping.

Proof. Let $\tilde{F}: K_{\alpha} \rightarrow \mathbb{F}_{0}$ be G-differentiable $\alpha$-preinvex fuzzy mapping. Since $\tilde{F}$ is $\alpha$-preinvex then, for each $u, y \in K_{\alpha}$ and $t \in[1,0]$, we have

$$
\begin{aligned}
\tilde{F}(u+\tau \alpha(y, u) \xi(y, u)) & \leq(1-\tau) \tilde{F}(u) \widetilde{\mp} \tau \tilde{F}(y), \\
& =\tilde{F}(u) \widetilde{+} \tau(\tilde{F}(y) \simeq \tilde{F}(u)),
\end{aligned}
$$

which implies that

$$
\begin{gathered}
\tau(\tilde{F}(y) \simeq \tilde{F}(u)) \geq \tilde{F}(u+\tau \alpha(y, u) \xi(y, u)) \simeq \tilde{F}(u), \\
\tilde{F}(y) \simeq \tilde{F}(u) \geq \frac{\tilde{F}(u+\tau \alpha(y, u) \xi(y, u)) \simeq \tilde{F}(u)}{\tau},
\end{gathered}
$$

taking limit in the above inequality as $\tau \rightarrow \mathbf{0}$, we have

$$
\tilde{F}(y) \simeq \tilde{F}(u) \geq\left\langle\alpha(y, u) \tilde{F}^{\prime}(u), \xi(y, u)\right\rangle .
$$

Conversely, let $\tilde{F}$ be a $\alpha$-invex fuzzy mapping. Since $K_{\alpha}$ is an $\alpha$-invex set so we have, $y_{\tau}=u+\tau \alpha(y, u) \xi(y, u) \in K_{\alpha}$ for all $u, y \in K_{\alpha}$ and $t \in[1,0]$. Taking $y=y_{\tau}$ in 
(3.7), we get

$$
\tilde{F}\left(y_{\tau}\right) \simeq \tilde{F}(u) \geq\left\langle\alpha\left(y_{\tau}, u\right) \tilde{F}^{\prime}(u), \xi\left(y_{\tau}, u\right)\right\rangle,
$$

using Condition $\mathrm{C}$, we have

$$
\tilde{F}\left(y_{\tau}\right) \simeq \tilde{F}(u) \geq(1-\tau) \alpha\left(y_{\tau}, u\right)\left\langle\tilde{F}^{\prime}(u), \xi(y, u)\right\rangle .
$$

In a similar way, we have

$$
\begin{aligned}
\tilde{F}(u) \simeq \tilde{F}\left(y_{\tau}\right) & \geq\left\langle\alpha\left(u, y_{\tau}\right) \tilde{F}^{\prime}(u), \xi\left(u, y_{\tau}\right)\right\rangle \\
& =-\tau \alpha\left(y, y_{\tau}\right)\left\langle\tilde{F}^{\prime}(u), \xi\left(y, y_{\tau}\right)\right\rangle,
\end{aligned}
$$

Multiplying (3.10) by $\tau$ and (3.11) by $(1-\tau)$, and adding the resultant, we have

$$
\tilde{F}\left(y_{\tau}\right) \preceq(1-\tau) \tilde{F}(u) \widetilde{+} \tau \tilde{F}(y),
$$

which implies that

$$
\tilde{F}(u+\tau \alpha(y, u) \xi(y, u)) \leq(1-\tau) \tilde{F}(u) \tilde{+} \tau \tilde{F}(y) .
$$

Hence, $\tilde{F}$ is $\alpha$-preinvex fuzzy mapping with respect to $\xi, \alpha$.

If $\alpha(y, u)=1$, then Theorem 4, collapse to the following result for preinvex (invex) fuzzy mappings.

Theorem 5. Let $\tilde{F}$ be a G-differentiable preinvex fuzzy mapping with respect to $\xi$ and let Condition D hold. Then $\tilde{F}$ is preinvex fuzzy mapping if and only if $\tilde{F}$ is invex fuzzy mapping.

Theorem 6. Let $\tilde{F}$ be a G-differentiable fuzzy mapping on $K_{\alpha}$. If the fuzzy mapping $\tilde{F}$ is $\alpha$-invex, then

$$
\left\langle\alpha(y, u) \tilde{F}^{\prime}(u), \xi(y, u)\right\rangle \widetilde{+}\left\langle\alpha(u, y) \tilde{F}^{\prime}(y), \xi(u, y)\right\rangle \preceq \tilde{0}, \text { for all } u, y \in K_{\alpha} .
$$

Conversely, if $\alpha(y, u)=\alpha(u, y)$, for all $u, y \in K_{\alpha}$, then $\tilde{F}$ is $\alpha$-invex fuzzy mapping with respect to $\xi, \alpha$ provided Conditions $A$ and $C$.

Proof. Let $\tilde{F}$ is $\alpha$-invex fuzzy mapping. Then,

$$
\tilde{F}(y) \simeq \tilde{F}(u) \geq\left\langle\alpha(y, u) \tilde{F}^{\prime}(u), \xi(y, u)\right\rangle \text {, for all } u, y \in K_{\alpha},
$$

replacing $y$ by $u$ and $u$ by $y$ in (3.13), we get

$$
\tilde{F}(u) \simeq \tilde{F}(y) \geq\left\langle\alpha(u, y) \tilde{F}^{\prime}(y), \xi(u, y)\right\rangle \text {, for all } u, y \in K_{\alpha} .
$$


Adding (3.13) and (3.14), we have

$$
\left\langle\alpha(y, u) \tilde{F}^{\prime}(u), \xi(y, u)\right\rangle \widetilde{f}\left\langle\alpha(u, y) \tilde{F}^{\prime}(y), \xi(u, y)\right\rangle \preceq \tilde{0} \text {, for all } u, y \in K_{\alpha} .
$$

Conversely, assume that (3.12) hold. Then

$$
\left\langle\alpha(y, u) \tilde{F}^{\prime}(u), \xi(y, u)\right\rangle \preceq \simeq\left\langle\alpha(u, y) \tilde{F}^{\prime}(y), \xi(u, y)\right\rangle, \text { for all } u, y \in K_{\alpha},
$$

which implies that

$$
\left\langle\tilde{F}^{\prime}(u), \xi(y, u)\right\rangle \leq \simeq\left\langle\tilde{F}^{\prime}(y), \xi(u, y)\right\rangle,
$$

since $\alpha(y, u)=\alpha(u, y)$.

Since $K_{\alpha}$ is an $\alpha$-invex set so we have, $y_{\tau}=u+\tau \alpha(y, u) \xi(y, u) \in K_{\alpha}$ for all $u, y \in K_{\alpha}$ and $t \in[1,0]$. Taking $y=y_{\tau}$ in (3.15), we get

$$
\left\langle\tilde{F}^{\prime}(u+\tau \alpha(y, u) \xi(y, u)), \xi(u, u+\tau \xi(y, u))\right\rangle \preceq \simeq\left\langle\tilde{F}^{\prime}(u), \xi(u+\tau \xi(y, u), u)\right\rangle,
$$

by using Condition $\mathrm{C}$, we have

$$
\begin{gathered}
\left\langle\tilde{F}^{\prime}(u+\tau \alpha(y, u) \xi(y, u)), \tau \xi(y, u)\right\rangle \geq\left\langle\tilde{F}^{\prime}(u), \tau \xi(y, u)\right\rangle, \\
\left\langle\tilde{F}^{\prime}(u+\tau \alpha(y, u) \xi(y, u)), \xi(y, u)\right\rangle \geq\left\langle\tilde{F}^{\prime}(u), \xi(y, u)\right\rangle .
\end{gathered}
$$

Let

$$
\tilde{h}(\tau)=\tilde{F}(u+\tau \alpha(y, u) \xi(y, u)),
$$

taking G-derivative with respect to $\tau$, we get

$$
\begin{aligned}
\tilde{h}^{\prime}(\tau) & =\tilde{F}^{\prime}(u+\tau \alpha(y, u) \xi(y, u)) \alpha(y, u) \xi(y, u) \\
& =\left\langle\alpha(y, u) \tilde{F}^{\prime}(u+\tau \alpha(y, u) \xi(y, u)), \xi(y, u)\right\rangle,
\end{aligned}
$$

from which, using (3.16), we have

$$
\tilde{h}^{\prime}(\tau) \geq\left\langle\alpha(y, u) \tilde{F}^{\prime}(u+\tau \alpha(y, u) \xi(y, u)), \xi(y, u)\right\rangle .
$$

Integrating (3.17) between 0 to1 with respect to $\tau$, we get

$$
\tilde{h}(1) \simeq \tilde{h}(0) \geq\left\langle\alpha(y, u) \tilde{F}^{\prime}(u), \xi(y, u)\right\rangle,
$$

which implies that

$$
\tilde{F}(u+\alpha(y, u) \xi(y, u)) \simeq \tilde{F}(u) \geq\langle\alpha(y, u) \tilde{F} \cdot(u), \xi(y, u)\rangle,
$$


By using Condition, A

$$
\tilde{F}(y) \simeq \tilde{F}(u) \geq\left\langle\alpha(y, u) \tilde{F}^{\prime}(u), \xi(y, u)\right\rangle \text {, for all } u, y \in K_{\alpha} .
$$

Showing that $\tilde{F}$ is $\alpha \xi$-invex fuzzy mapping on $K_{\alpha}$.

Definition 22. A G-differentiable fuzzy mapping $\tilde{F}: K_{\alpha} \rightarrow \mathbb{F}_{0}$ is said to be:

(i) $\alpha \xi$-monotone operator with respect to $\xi, \alpha$ if and only if,

$$
\left\langle\alpha(y, u) \tilde{F}^{\prime}(u), \xi(y, u)\right\rangle \widetilde{+}\left\langle\alpha(u, y) \tilde{F}^{\prime}(y), \xi(u, y)\right\rangle \leq \tilde{0} \text {, for all } u, y \in K_{\alpha} .
$$

(ii) $\alpha \xi$-pseudomonotone operator with respect to $\xi, \alpha$ if and only if,

$$
\left\langle\alpha(y, u) \tilde{F}^{\prime}(u), \xi(y, u)\right\rangle \geq \tilde{0} \Rightarrow \simeq\left\langle\alpha(u, y) \tilde{F}^{\prime}(y), \xi(u, y)\right\rangle \geq \tilde{0} \text {, for all } u, y \in K_{\alpha} .
$$

(iii) Strictly $\alpha \xi$-monotone operator with respect to $\xi, \alpha$ if and only if,

$$
\left\langle\alpha(y, u) \tilde{F}^{\prime}(u), \xi(y, u)\right\rangle \tilde{+}\left\langle\alpha(y, u) \tilde{F}^{\prime}(y), \xi(u, y)\right\rangle \prec \tilde{0} \text {, for all } u, y \in K_{\alpha} .
$$

(iv) quasi $\alpha \xi$-monotone operator with respect to $\xi, \alpha$ if and only if,

$$
\left\langle\alpha(y, u) \tilde{F}^{\prime}(u), \xi(y, u)\right\rangle>\tilde{0} \Rightarrow\left\langle\alpha(y, u) \tilde{F}^{\prime}(y), \xi(u, y)\right\rangle \leq \tilde{0} \text {, for all } u, y \in K_{\alpha} .
$$

(v) Strictly $\alpha \xi$-pseudomonotone operator with respect to $\xi, \alpha$ if and only if,

$$
\left\langle\alpha(y, u) \tilde{F}^{\prime}(u), \xi(y, u)\right\rangle \geq \tilde{0} \Rightarrow\left\langle\alpha(y, u) \tilde{F}^{\prime}(y), \xi(u, y)\right\rangle \prec \tilde{0} \text {, for all } u, y \in K_{\alpha} .
$$

If $\xi(y, u)=-\xi(u, y)$, then Definition 22, reduce to new one.

If $\tilde{F}$, is $\alpha \xi$-monotone operator, then Theorem 6 can also be written as:

Theorem 7. Let $\tilde{F}$ be a G-differentiable fuzzy mapping on the $K_{\alpha}$ and let Conditions $A$ and $C$ hold. If $\alpha(y, u)=\alpha(u, y)$, then the fuzzy mapping $\tilde{F}$ is $\alpha$-invex ( $\alpha$-preinvex) if and only if, its $G$-differential $\tilde{F}^{\prime} \alpha \xi$-monotone operator.

If $\alpha(y, u)=1$, then Theorem 7 collapse to the following result for preinvex (invex) fuzzy mappings.

Theorem 8. Let $\tilde{F}$, be a G-differentiable fuzzy mapping of $\tilde{F}$ on $K_{\alpha}$ and let Conditions $B$ and D hold. Then $\tilde{F}$, is $\xi$-monotone operator if and only if, $\tilde{F}$ is invex fuzzy mapping.

As special cases of Theorem 10, we have the following

Theorem 9. Let G-differential $\tilde{F}$ ' of mapping $\tilde{F}$ on $K_{\alpha}$ be $\alpha \xi$-pseudomonotone operator and let Conditions $A$ and $C$. Then $\tilde{F}$ is a pseudo $\alpha \xi$-invex fuzzy mapping. 
Proof. Let $\tilde{F}^{\prime}$ be $\alpha \xi$-pseudomonotone operator. Then for all $u, y \in K_{\alpha}$,

$$
\left\langle\alpha(y, u) \tilde{F}^{\prime}(u), \xi(y, u)\right\rangle \geq \tilde{0},
$$

which implies that

$$
\simeq\left\langle\alpha(u, y) \tilde{F}^{\prime}(y), \xi(u, y)\right\rangle \geq \tilde{0}
$$

Since $K_{\alpha}$ is an $\alpha$-invex set then, we have, $y_{\tau}=u+\tau \alpha(y, u) \xi(y, u) \in K_{\alpha}$ for all $u, y \in K_{\alpha}$ and $t \in[1,0]$. Taking $y=y_{\tau}$ in (3.18), we get

$$
\simeq \alpha\left(u, y_{\tau}\right)\left\langle\tilde{F}^{\prime}(u+\tau \alpha(y, u) \xi(y, u)), \xi(u, u+\tau \xi(y, u))\right\rangle \geq \tilde{0},
$$

by using Condition $\mathrm{C}$, we have

$$
\left\langle\tilde{F}^{\prime}(u+\tau \alpha(y, u) \xi(y, u)), \xi(y, u)\right\rangle \geq \tilde{0} .
$$

Let

$$
\tilde{h}(\tau)=\tilde{F}(u+\tau \alpha(y, u) \xi(y, u)),
$$

taking G-derivative with respect to $\tau$, we get

$$
\tilde{h}^{\prime}(\tau)=\left\langle\alpha(y, u) \tilde{F}^{\prime}(u+\tau \alpha(y, u) \xi(y, u)), \xi(y, u)\right\rangle,
$$

from which, using (3.19), we have

$$
\tilde{h}^{\prime}(\tau) \geq \tilde{0} .
$$

Integrating (3.20) between 0 to 1 with respect to $\tau$, we get

$$
\tilde{h}(1) \simeq \tilde{h}(0) \geq \tilde{0},
$$

which implies that

$$
\tilde{F}(u+\alpha(y, u) \xi(y, u)) \simeq \tilde{F}(u) \geq \tilde{0},
$$

by using Condition, A

$$
\tilde{F}(y) \simeq \tilde{F}(u) \geq \tilde{0} \text {, for all } u, y \in K_{\alpha} .
$$

Showing that $\tilde{F}$ is a pseudo $\alpha \xi$-invex mapping on the $\alpha$-invex set $K_{\alpha}$.

Theorem 10. Let $G$-differential $\tilde{F}$ ' of mapping $\tilde{F}$ on $K_{\alpha}$ be quasi $\alpha \xi$-monotone operator, and let Conditions $A$ and $C$. Then $\tilde{F}$ is a quasi $\alpha \xi$-invex mapping.

Proof. The demonstration is analogous to the demonstration of Theorem 9. 


\section{Applications}

We now discuss the fuzzy optimality condition for $\alpha$-preinvex fuzzy mappings, which is main motivation of our next results.

Theorem 11. Let $\tilde{F}: K_{\alpha} \rightarrow \mathbb{F}_{0}$ be a fuzzy mapping, with $\tilde{\sigma}=i n f_{u \in K_{\alpha}}(\tilde{F}(u))$ exists in $\mathbb{F}_{0}$.

(1) If $\tilde{F}: K_{\alpha} \rightarrow \mathbb{F}_{0}$ is a $\alpha$-preinvex fuzzy mapping on $K_{\alpha}$, then set $\$=\left\{u: u \in K_{\alpha}\right.$, $\tilde{F}(u)=\tilde{\sigma}\}$ is a $\alpha$-invex set.

(2) If $\tilde{F}: K_{\alpha} \rightarrow \mathbb{F}_{0}$ is a strictly $\alpha$-preinvex fuzzy mapping on $K_{\alpha}$, then $\$$ is a singleton set or empty. That is, if $\tilde{F}$ is strictly $\alpha$-preinvex, then $\tilde{F}$ has at least one global minimum.

Proof. (1) Let $\tilde{F}$ be $\alpha$-preinvex fuzzy mapping. If $\$$ is an empty set, then is an $\alpha$-invex set. Assume that, $u, y \in \$$, that is $u, y \in K_{\alpha}$ and $\tilde{F}(u)=\widetilde{\sigma}=\tilde{F}(y)$. Since $\tilde{F}$ is $\alpha$-preinvex fuzzy mapping,

$$
\begin{aligned}
\tilde{F}(u+\tau \alpha(y, u) \xi(y, u)) & \leq(1-\tau) \tilde{F}(u) \widetilde{+} \tau \tilde{F}(y), \\
& =(1-\tau) \widetilde{\sigma} \widetilde{+} \tau \widetilde{\sigma}=\widetilde{\sigma},
\end{aligned}
$$

for all $\tau \in(0,1)$. Hence, all points of the form $u+\tau \alpha(y, u) \xi(y, u) \in \$, \tau \in[0,1]$ which implies that $\$$ is an $\alpha$-invex set.

(2) Let $\tilde{F}$ be a strictly $\alpha$-preinvex fuzzy mapping on $K_{\alpha}$. Contrary suppose that there exist distinct points $u, y \in \$$ such that $\tilde{F}(u)=\widetilde{\sigma}=\tilde{F}(y)$. Since $\tilde{F}$ is strictly $\alpha$-preinvex fuzzy mapping then, for all $u, y \in K_{\alpha}$ and $\tau \in(0,1)$, we have

$$
\begin{aligned}
\tilde{F}(u+\tau \alpha(y, u) \xi(y, u)) & \prec(1-\tau) \tilde{F}(u) \widetilde{+} \tau \tilde{F}(y), \\
& =(1-\tau) \widetilde{\sigma} \widetilde{+} \widetilde{\sigma}=\widetilde{\sigma},
\end{aligned}
$$

which implies that $\widetilde{\sigma} \neq \inf _{u \in K_{\alpha}}(\tilde{F}(u))$, so this contradict the fact. Hence, the result follows.

Theorem 12. Let $K_{\alpha}$ be a nonempty set. Suppose that $\tilde{F}: K_{\alpha} \rightarrow \mathbb{F}_{0}$ be a $\alpha$-preinvex fuzzy mapping on $K_{\alpha}$, with $\tilde{\sigma}=i n f_{u \in K_{\alpha}}(\tilde{F}(u))$ exists in $[0,1]$, and that the set

$$
\$=\left\{u: u \in K_{\alpha}, \tilde{F}(u)=\tilde{\sigma}\right\} \neq \emptyset .
$$

If $\tilde{u}$ is local minimum of $\tilde{F}$, then it is also a global minimum of $\tilde{F}$ on $K_{\alpha}$. 
Proof. Let $\tilde{F}: K_{\alpha} \rightarrow \mathbb{F}_{0}$ be a $\alpha$-preinvex fuzzy mapping and $u$ is local minimum of $\tilde{F}$. If $u$ is not global minimum of $\tilde{F}$, then $u \notin \$$. By hypothesis, $\$ \neq \emptyset$. If $y \in \$$, then we must have $\tilde{F}(y) \prec \tilde{F}(u)$. Since $\tilde{F}$ is $\alpha$-preinvex then, for $\tau \in(1,0)$, we have

$$
\tilde{F}(u+\tau \alpha(y, u) \xi(y, u)) \preccurlyeq(1-\tau) \tilde{F}(u) \widetilde{F} \tilde{F}(y) \prec \tilde{F}(u) \text {, because } \tilde{F}(y) \prec \tilde{F}(u),
$$

for any small positive number $\tau$, and this contradiction proves the required result.

Theorem 13. Let $\tilde{F}: K_{\alpha} \rightarrow \mathbb{F}_{0}$ be a quasi $\alpha$-preinvex fuzzy mapping on $K_{\alpha}$. If $u \in K_{\alpha}$ is a strict local minimum of $\tilde{F}$, then it is also a strict global minimum of $\tilde{F}$ on $K_{\alpha}$.

Proof. Let $\tilde{u} \in K_{\alpha}$ is a strict local minimum of $\tilde{F}$. Then by definition of strict local minimum, there exist a $\delta>0$ such that $\tilde{F}(u) \prec \tilde{F}(y)$ for all $y \in K_{\alpha} \cap B_{\delta}(u)$. Assume contrary, that is, for some $y^{\prime} \in K_{\alpha}$, we have $\tilde{F}(u) \geqslant \tilde{F}\left(y^{\prime}\right)$. Since $\tilde{F}$ is a quasi $\alpha$-preinvex fuzzy mapping then, for $\tau \in(1,0)$, we have

$$
\tilde{F}(u+\tau \alpha(y, u) \xi(y, u)) \preccurlyeq \max (\tilde{F}(u), \tilde{F}(y))=\tilde{F}(u) \text {, because } \tilde{F}(u) \succcurlyeq \tilde{F}\left(y^{\prime}\right) .
$$

This contradicts that $u \in K_{\alpha}$ is a strict local minimum of $\tilde{F}$, and hence the results follows.

Theorem 14. Let $\tilde{F}$ be a G-differentiable and $\alpha$-preinvex mapping with respect to $\xi, \alpha$. Then $u \in K_{\alpha}$ is the minimum of the mapping $\tilde{F}$, if and only if, $u \in K_{\alpha}$ satisfies

$$
\left\langle\alpha(y, u) \tilde{F}^{\prime}(u), \xi(y, u)\right\rangle \geq \tilde{0}, \text { for all } y \in K_{\alpha},
$$

with respect to $\xi, \alpha$.

Proof. Let $u \in K_{\alpha}$ be a minimum of the $\alpha$-preinvex fuzzy mapping $\tilde{F}$. Then for all $y \in K_{\alpha}$, we have

$$
\tilde{F}(y) \geq \tilde{F}(u),
$$

since $K_{\alpha}$ is an $\alpha$-invex set then, we have

$$
y_{\tau}=u+\tau \alpha(y, u) \xi(y, u) \in K_{\alpha} \text { for all } u, y \in K_{\alpha} \text { and } t \in[1,0] .
$$

Taking $y=y_{\tau}$ in (4.2), we get

$$
\tilde{F}(u+\tau \alpha(y, u) \xi(y, u)) \simeq \tilde{F}(u) \geq \tilde{0},
$$

dividing the above the inequality by $\tau$, we have

$$
\frac{\tilde{F}(u+\tau \alpha(y, u) \xi(y, u)) \simeq \tilde{F}(u)}{\tau} \geq \tilde{0},
$$


taking limit in the above inequality as $\tau \rightarrow \mathbf{0}$, we get

$$
\langle\alpha(y, u) \tilde{F},(u), \xi(y, u)\rangle \geq \tilde{0} .
$$

The required (4.1), since $\tilde{F}$ is a G-differentiable fuzzy mapping.

Conversely, let $u \in K_{\alpha}$ satisfies (4.1). Since $\tilde{F}$ is a G-differentiable $\alpha$-preinvex fuzzy mapping, then by (3.7), we have

$$
\tilde{F}(y) \simeq \tilde{F}(u) \geq\langle\tilde{F},(u), \alpha(y, u) \xi(y, u)\rangle,
$$

from which, using (21), we have

$$
\tilde{F}(y) \simeq \tilde{F}(u) \geq 0
$$

which implies that

$$
\tilde{F}(y) \geq \tilde{F}(u) \text {, for all } y \in K_{\alpha} .
$$

Showing that $u \in K_{\alpha}$ is the minimum of the mapping, the required result.

For G-differentiable $\alpha$-invex fuzzy mapping, we have the following result.

Theorem 15. If $\tilde{F}: K_{\alpha} \rightarrow \mathbb{F}_{0}$ be a G-differentiable $\alpha$-invex fuzzy mapping with respect to $\xi, \alpha$. If $u \in K_{\alpha}$ is the minimum of the mapping $\widetilde{F}$ if and only if $u \in K_{\alpha}$ satisfies (4.1).

Remark 3. The inequality of the type (4.1) is called fuzzy $\alpha$-variational-like inequality. We would like to emphasize that, when $\alpha(y, u)=1$, then optimality conditions of the $\alpha$-preinvex fuzzy mapping can be characterized by the following inequality,

$$
\left\langle\tilde{F}^{\prime}(u), \xi(y, u)\right\rangle \geq \tilde{0}, \text { for all } u, y \in K_{\alpha},
$$

which is called fuzzy variational-like inequality. For application of variational-like inequalities and fuzzy variation-like inequalities, see [14, 15, 16, 17, 18, 19, 20, 21, 23].

\section{Conclusion}

In this paper, we have introduced and studied new class of non-convex fuzzy mappings with respect to bi-functions $\xi, \alpha$. Which is called $\alpha$-preinvex fuzzy mappings with respect to $\xi, \alpha$. We have investigated characterization of preinvex fuzzy mappings in term of $\alpha$-preinvex fuzzy mappings. It is shown that convex and preinvex fuzzy mappings are special cases of $\alpha$-preinvex fuzzy mappings. We have proposed several new concepts of $\xi$-invex fuzzy mappings and $\alpha \xi$-monotonicities, and then discuss their 
relation. It is proved that minimum of $\alpha$-preinvex mappings can be characterized by $\alpha$-varitional like inequalities and varitional like inequalities. One can obtain important and significant applications in generalized convex fuzzy programming and multiobjective fuzzy optimization, see $[10,16]$.

\section{References}

[1] B. Bede and S. G. Gal, Generalizations of the differentiability of fuzzy-number-valued functions with applications to fuzzy differential equations, Fuzzy Sets and Systems 151(3) (2005), 581-599. https://doi.org/10.1016/j.fss.2004.08.001

[2] A. Ben-Israel and B. Mond, What is invexity?, The ANZIAM Journal 28 (1986), 1-9. https://doi.org/10.1017/S0334270000005142

[3] J. Cervelati, M. D. Jiménez-Gamero, F. Vilca-Labra and M. A. Rojas-Medar, Continuity for s-convex fuzzy processes, in: Soft Methodology and Random Information Systems, Advances in Soft Computing, vol. 26, Springer, Berlin, Heidelberg, 2004, pp. 653-660. https://doi.org/10.1007/978-3-540-44465-7_81

[4] Y. Chalco-Cano, M. A. Rojas-Medar and H. Román-Flores, $M$-convex fuzzy mappings and fuzzy integral mean, Comput. Math. Appl. 40(10-11) (2000), 1117-1126. https://doi.org/10.1016/S0898-1221(00)00226-1

[5] S. S. L. Chang and L. A. Zadeh, On fuzzy mapping and control, IEEE Trans. Systems Man Cybernet. 2 (1972), 30-34.

https://doi.org/10.1109/TSMC.1972.5408553

[6] X. P. Ding and J. Y. Park, A new class of generalized nonlinear implicit quasivariational inclusions with fuzzy mappings, J. Comput. Appl. Math. 138(2) (2002), 243-257. https://doi.org/10.1016/S0377-0427(01)00379-X

[7] N. Furukawa, Convexity and local Lipschitz continuity of fuzzy-valued mappings, Fuzzy Sets and Systems 93(1) (1998), 113-119. https://doi.org/10.1016/S0165-0114(96)00192-3

[8] R. Goetschel, Jr. and W. Voxman, Elementary fuzzy calculus, Fuzzy Sets and Systems 18(1) (1986), 31-43. https://doi.org/10.1016/0165-0114(86)90026-6

[9] M. A. Hanson, On sufficiency of the Kuhn-Tucker conditions, J. Math. Anal. Appl. 80 (1981), 545-550. https://doi.org/10.1016/0022-247X(81)90123-2

[10] V. Jeyakumar and B. Mond, On generalized convex mathematical programming, The ANZIAM Journal 34 (1992), 43-53. https://doi.org/10.1017/S0334270000007372

[11] J. Li and M. A. Noor, On characterizations of preinvex fuzzy mappings, Comput. Math. Appl. 59(2) (2010), 933-940. https://doi.org/10.1016/j.camwa.2009.09.015 
[12] S. R. Mohan and S. K. Neogy, On invex sets and preinvex functions, J. Math. Anal. Appl. 189 (1995), 901-908. https://doi.org/10.1006/jmaa.1995.1057

[13] S. Nanda and K. Kar, Convex fuzzy mappings, Fuzzy Sets and Systems 48(1) (1992), 129-132. https://doi.org/10.1016/0165-0114(92)90256-4

[14] M. A. Noor, Fuzzy preinvex functions, Fuzzy Sets and Systems 64(1) (1994), 95-104. https://doi.org/10.1016/0165-0114(94)90011-6

[15] M. A. Noor, Variational-like inequalities, Optimization 30 (1994), 323-330. https://doi.org/10.1080/02331939408843995

[16] M. A. Noor and K. I. Noor, Some characterizations of strongly preinvex functions, $J$. Math. Anal. Appl. 316(2) (2006), 697-706. https://doi.org/10.1016/j.jmaa.2005.05.014

[17] M. A. Noor, Variational inequalities for fuzzy mappings (III), Fuzzy Sets and Systems 110(1) (2000), 101-108. https://doi.org/10.1016/S0165-0114(98)00131-6

[18] M. A. Noor, Generalized convex functions, Panamer. Math. J. 4 (1994), 73-89.

[19] A. Rufián-Lizana, Y. Chalco-Cano, R. Osuna-Gómez and G. Ruiz-Garzón, On invex fuzzy mappings and fuzzy variational-like inequalities, Fuzzy Sets and Systems 200 (2012), 84-98. https://doi.org/10.1016/j.fss.2012.02.001

[20] A. Rufián-Lizana, Y. Chalco-Cano, G. Ruiz-Garzón and H. Román-Flores, On some characterizations of preinvex fuzzy mappings, TOP 22(2) (2014), 771-783. https://doi.org/10.1007/s11750-013-0299-3

[21] L. Stefanini and B. Bede, Generalized Hukuhara differentiability of interval-valued functions and interval differential equations, Nonlinear Anal. 71(3-4) (2009), 1311-1328. https://doi.org/10.1016/j.na.2008.12.005

[22] Y. R. Syau, Preinvex fuzzy mappings, Comput. Math. Appl. 37(3) (1999), 31-39. https://doi.org/10.1016/S0898-1221(99)00044-9

[23] Y. R. Syau, Invex and generalized convex fuzzy mappings, Fuzzy Sets and Systems 115(3) (2000), 455-461. https://doi.org/10.1016/S0165-0114(98)00415-1

[24] Y. R. Syau, Generalization of preinvex and B-vex fuzzy mappings, Fuzzy Sets and Systems 120(3) (2001), 533-542. https://doi.org/10.1016/S0165-0114(99)00139-6

[25] Y. R. Syau, $\left(\Phi_{1}, \Phi_{2}\right)$-convex fuzzy mappings, Fuzzy Sets and Systems 138(3) (2003) 617 625. https://doi.org/10.1016/S0165-0114(02)00527-4

[26] Y. R. Syau and E. S. Lee, Fuzzy Weirstrass theorem and convex fuzzy mappings, Comput. Math. Appl. 51(12) (2006), 1741-1750.

https://doi.org/10.1016/j.camwa.2006.02.005 
[27] Y. R. Syau, On convex and concave fuzzy mappings, Fuzzy Sets and Systems 103(1) (1999), 163-168. https://doi.org/10.1016/S0165-0114(97)00210-8

[28] T. Weir and B. Mond, Pre-invex functions in multiple objective optimization, J. Math. Anal. Appl. 136 (1988), 29-38. https://doi.org/10.1016/0022-247X(88)90113-8

[29] Z. Wu and J. Xu, Generalized convex fuzzy mappings and fuzzy variational-like inequality, Fuzzy Sets and Systems 160(11) (2009), 1590-1619. https://doi.org/10.1016/j.fss.2008.11.031

[30] X. Q. Yang and G. Y. Chen, A class of nonconvex functions and variational inequalities, J. Math. Anal. Appl. 169 (1992), 359-373. https://doi.org/10.1016/0022-247X(92)90084-Q

[31] H. Yan and J. Xu, A class of convex fuzzy mappings, Fuzzy Sets and Systems 129(1) (2002), 47-56. https://doi.org/10.1016/S0165-0114(01)00157-9

This is an open access article distributed under the terms of the Creative Commons Attribution License (http://creativecommons.org/licenses/by/4.0/), which permits unrestricted, use, distribution and reproduction in any medium, or format for any purpose, even commercially provided the work is properly cited.. 